Artículo Original

\title{
Manejo de la broca del café, Hypothenemus hampei (Ferrari, 1867) (Coleoptera: Curculionidae: Scolytinae), con atrayentes etanólicos en cultivos de café de Coatepec, Veracruz, México
}

\author{
Management of the coffee borer beetle, Hypothenemus hampei (Ferrari, 1867) (Coleoptera: \\ Curculionidae: Scolytinae), with ethanolic attractants in coffee cultivation of Coatepec, \\ Veracruz, Mexico
}

Guillermo Mendoza-Cervantes ${ }^{1}{ }^{(D)}$, Oswaldo Guzmán-López ${ }^{2}$ (D) y Alejandro Salinas-Castro ${ }^{1^{*}}$ (D)

${ }^{1}$ Centro de Investigación en Micología Aplicada, Universidad Veracruzana, Calle Médicos 5, Colonia Unidad del Bosque, C.P. 91010, Xalapa, Veracruz, México. ${ }^{2}$ Facultad de Ciencias Químicas, Universidad Veracruzana campus Coatzacoalcos, Av. Universidad Km 7.5, Col. Santa Isabel, C.P. 96535. Coatzacoalcos, Veracruz, México.良*asalinas@uv.mx

\section{ZooBank: urn:lsid:zoobank.org:pub:8CEA35E5-E12D-4CC4-B1A0-7BDE8DE8AA2E https: / / doi.org/ 10.35249/rche.47.2.21.14}

Resumen. La broca del café Hypothenemus hampei, es uno de los insectos plaga más importantes a nivel mundial que dañan al fruto del café. El objetivo fue evaluar vinagres y extractos de plantas mezclados con etanol para ser utilizados como atrayentes para la captura del insecto. Se utilizaron por primera vez las mezclas: etanol-albahaca, etanol-ruda, etanol-romero, etanolvinagre blanco y etanol-vinagre de manzana, en una proporción 1:1 y como testigo metanol-etanol 3:1, utilizando trampas artesanales, en un diseño completamente al azar con cinco repeticiones por cada tratamiento. Con base en los análisis estadísticos, se observó que el etanol-vinagre de manzana y etanol-vinagre blanco, presentaron la mayor captura de especímenes 136,36 y 98,24 en promedio con respecto a las otras mezclas. Se concluye que los mejores atrayentes utilizados en esta investigación pueden servir como una estrategia sustentable para la captura de la broca del café.

Palabras clave: Atrayentes; broca; mezclas; sustentable.

\begin{abstract}
The coffee borer beetle Hypothenemus hampei is one of the most important pest insects worldwide that cause damage to coffee berries. The objective was to evaluate vinegars and plant extracts mixed with ethanol to be used as attractants for the capture of coffee borer beetles. The following mixtures were used for the first time: ethanol-basil, ethanol-rue, ethanol-rosemary, ethanol-white vinegar, and ethanol-apple vinegar, in a proportion of 1:1 and as a control, the methanol-ethanol mixture 3:1 using handmade traps in a completely randomized design with five repetitions per treatment. Based on the statistical analyses, we observed that ethanol-apple vinegar and ethanol-white vinegar resulted in the highest capture of specimens, with a mean of 136.36 and 98.24, respectively, compared to the other mixtures. In conclusion, the most efficient attractants in this study could be used as a sustainable strategy for the capture of coffee borer beetles.
\end{abstract}

Key words: Attractants; borer beetle; mixtures; sustainable.

Recibido 12 Abril 2021 / Aceptado 5 Mayo 2021 / Publicado online 31 Mayo 2021 Editor Responsable: Belyani Vargas Batis 


\section{Introducción}

La broca del café, Hypothenemus hampei (Ferrari, 1867) (Coleoptera: Curculionidae: Scolytinae), es uno de los insectos plaga más importantes en las plantaciones de café (Coffea arabica L.) que se ha estudiado por más de un siglo (Green et al. 2015; Vega et al. 2015a). Este insecto vive dentro del fruto del café, donde las hembras construyen sus galerías en el endospermo, depositan sus huevos y cuando emergen se aparean y buscan otra baya para ovopositar (Rodríguez et al. 2017; Alba-Alejandre et al. 2018). El daño causado por la hembra y su progenie afecta la producción de café al causar pérdidas en el rendimiento y la calidad (Gómez et al. 2015; Infante 2018). Desafortunadamente las estrategias de manejo implementadas para la broca no funcionan de manera eficiente debido a que el insecto pasa gran parte de su ciclo biológico dentro de los frutos (Aristizábal et al. 2016).

Los productores de café con frecuencia utilizan insecticidas sintéticos para controlar a H. hampei, como el Endosulfán y el Clorpirifos, los cuales son altamente tóxicos para el ambiente, causando daños a la salud de los productores y además se ven afectadas las comunidades aledañas a las plantaciones de café (Cruz y Malo 2013; Infante 2018). El control biológico de esta plaga ha tenido bajo impacto cuando se ha evaluado con parasitoides importados de otros países de América Latina (Rodríguez et al. 2017). Por otra parte, para el control de H. hampei se ha evaluado en distintos lugares a los hongos entomopatógenos Beauveria bassiana (Bals.-Criv.) Vuill., 1912 y Paecilomyces fumosoroseus (Wize) A.H.S. Br. \& G. Sm. (1957), así como, nemátodos entomopatógenos que ha tenido un efecto limitado (Messing 2012).

Uno de los métodos de manejo más antiguos de H. hampei es el cultural, que es efectivo y ambientalmente ventajoso, pero esta práctica aumenta considerablemente los costos de producción, debido a que se recurre a la eliminación y destrucción de los granos de café infestados para reducir los niveles de la población; este método si puede funcionar en cualquier etapa del desarrollo de la fruta del café (Infante 2018).

Una alternativa que ya es común y ha dado buenos resultados, es el uso de trampas cebadas con semioquímicos, método que es utilizado en muchos países para el manejo de la H. hampei (Messing 2012; Pereira et al. 2012). La utilidad de semioquímicos para la broca del café se demostró a finales de la década de 1960, cuando Prates (1969) observó que con extractos de bayas de café verdes y maduras atraían a los adultos, probablemente debido a una combinación de señales visuales del insecto y de sustancias químicas del fruto (Mathieu et al. 2001; Bruce et al. 2005; Mendesil et al. 2009). En México se han utilizado trampas cebadas para capturar $\mathrm{H}$. hampei con mezclas sinérgicas de metanol: etanol $\left(\mathrm{CH}_{3} \mathrm{OH}: \mathrm{CH}_{3} \mathrm{CH}_{2} \mathrm{OH}\right)$ en proporción 3:1, respectivamente, debido a que son atractivos para la hembra apareada en condiciones de campo, sin embargo, se ha reportado que el uso de metanol puede resultar tóxico para los agricultores (Fernández y Cordero 2005; Aristizábal et al. 2015; Vega et al. 2015a, 2015b).

De esta manera, el presente trabajo tuvo como objetivo evaluar vinagres y extractos de plantas mezclados con etanol para ser utilizados como atrayentes para la captura del insecto.

\section{Materiales y Métodos}

Sitio de muestreo. El muestreo se realizó en una superficie aproximada de 1,5 ha en la comunidad de Bella Esperanza, del Municipio de Coatepec, Veracruz, con la ubicación geográfica $19^{\circ} 25^{\prime} 38,18^{\prime \prime} \mathrm{N}$ y $96^{\circ} 52^{\prime} 07,87^{\prime \prime} \mathrm{O}$ a $1297 \mathrm{msnm}$, con temperaturas que oscilan entre los $19{ }^{\circ} \mathrm{C}$ y $24^{\circ} \mathrm{C}$, humedad relativa del $69 \%$ y precipitación pluvial anual de 1798 $\mathrm{mm}$. Las fincas tienen plantas de café de la variedad garnica, geisha y Colombia con una edad de plantación de 5 años aproximadamente. La distancia entre plantas es de 1,80 m entre hileras y 1,2 m entre plantas, con un sistema de cultivo bajo sombra. 
Muestreo. Durante los meses de septiembre a diciembre de 2016 se realizaron los muestreos, extendiéndose ese año la cosecha en la región. Se usaron trampas cebadas con atrayentes para la captura de los insectos como está establecido por el Servicio Nacional de Sanidad e Inocuidad Agroalimentaria [SENASICA] (2008) en el muestreo de la broca, se colocaron botellas de PET de 2 L con tres ventanas y con un gotero de plástico de $10 \mathrm{ml}$ en su interior, como difusor del atrayente (Fig. 1). Al fondo de la trampa se colocó agua jabonosa como medio de captura. Por cada hectárea se colocaron 25 trampas distribuidas en forma de zig-zag, fijadas a las plantas de café a una altura de 1,5 m sobre el nivel del suelo, empleando alambre galvanizado del número 12 (SENASICA 2008). Con la finalidad de ver la presencia de la broca en los cafetales de la zona, se realizó un muestreo previo utilizando trampas con una mezcla de metanol: etanol como atrayente a una proporción 3:1 y posteriormente se realizaron los muestreos con los tratamientos del diseño experimental.

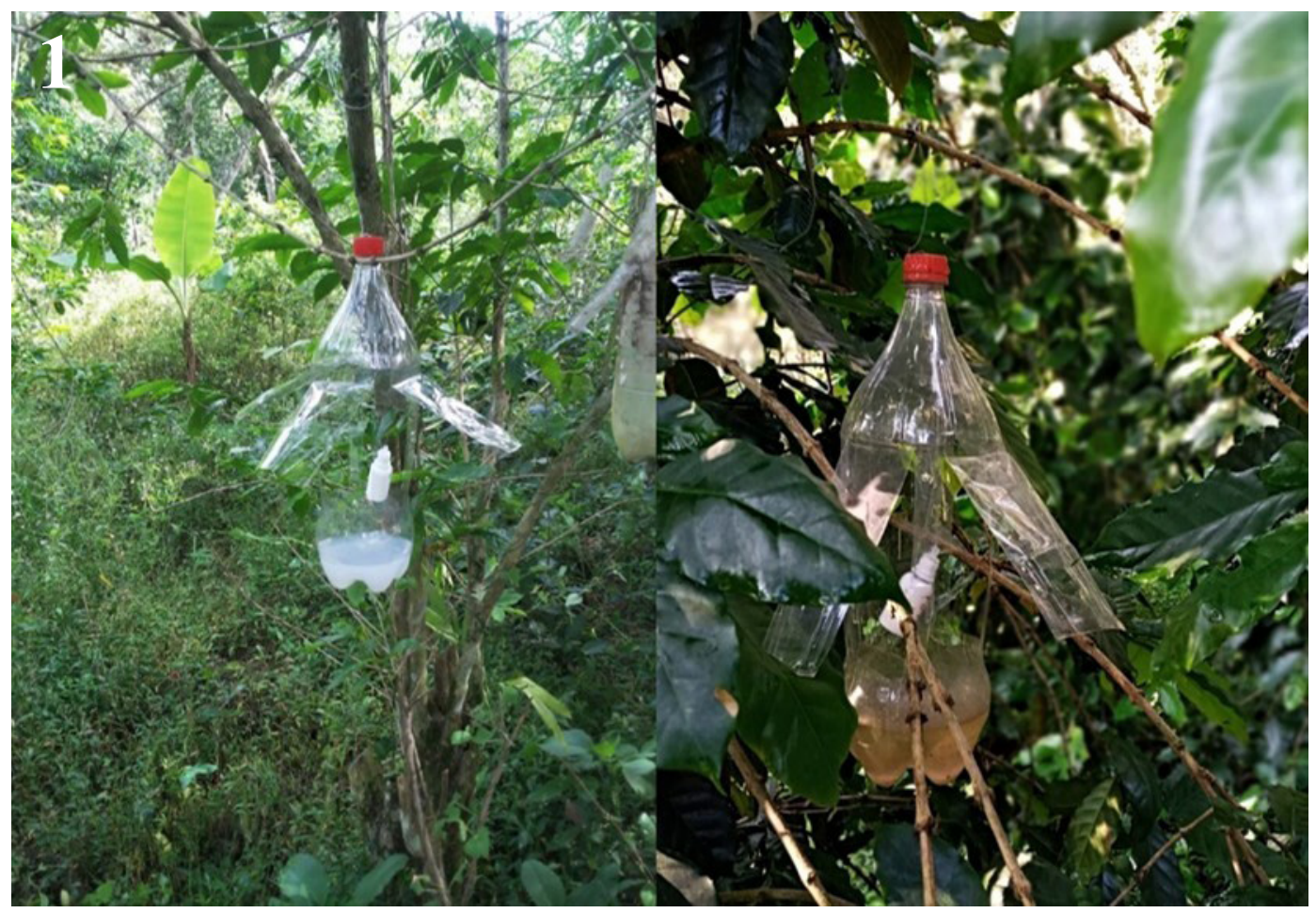

Figura 1. Trampas con atrayente para capturar adultos de H. hampei en plantas de café. / Handmade traps with attractants to capture $H$. hampei adults in coffee plants.

Obtención de los extractos etanólicos de las plantas. Las plantas de albahaca (Ocimum basilicum L.), ruda (Ruta graveolens L.) y romero (Rosmarinus officinalis L.) fueron obtenidas del Huerto Agroecológico de la Facultad de Ciencias Agrícolas en la Universidad Veracruzana, Veracruz, México; con un año de edad aproximadamente. Se tomaron partes blandas (hojas) de cada una de las plantas y se deshidrataron a $40 \pm 1{ }^{\circ} \mathrm{C}$ por 48 horas en un horno de secado; posteriormente, se fragmentaron en trozos pequeños y se colocaron por separado en un frasco de vidrio con tapa donde se maceraron con etanol en proporción 1:2 ( $\mathrm{p} / \mathrm{v})$ por 72 horas a temperatura ambiente. A continuación, los extractos se filtraron, concentraron en un evaporador rotario y se almacenaron hasta su uso como atrayentes. 
Diseño experimental. Se estableció un diseño completamente al azar con cinco tratamientos y cinco repeticiones. De esta manera, se evaluaron seis mezclas de atrayentes en proporción 1:1 (v/v): etanol-extracto de albahaca, etanol-extracto de ruda, etanolextracto de romero, etanol-vinagre blanco, etanol-vinagre de manzana y como testigo la muestra metanol: etanol 3:1, las cuales se colocaron en las trampas. Cada 8 días se supervisó la integridad de las trampas y cada quince días fue cambiando el contenido del gotero, y cada dos meses se cambiaron las trampas en mal estado. Los insectos capturados se recolectaron cada quince días, los cuales se etiquetaron y se llevaron al laboratorio para su cuantificación e identificación.

Identificación de los adultos de H. hampei. Se identificaron taxonómicamente los insectos recolectados, realizando observaciones en un microscopio estereoscópico Carls Zeiss y en microscopio compuesto en campo claro (10X). Se observó el número de asperidades en la parte anterior del pronoto, el septum en las antenas y el número de estrías e interestrías en los élitros, así como, el número de espinas en las tibias de las patas anteriores, esto con la finalidad de diferenciarla de la falsa broca (Vega et al. 2015a; Alba-Alejandre et al. 2019).

Análisis de datos. Los análisis estadísticos se llevaron a cabo con el programa R, se realizó un ANOVA para determinar la significancia entre atrayente, sitio y fecha de muestreo. Además, se llevó a cabo una prueba de Tukey $(\mathrm{p}=0,05)$ para realizar la comparación de medias entre los tratamientos.

\section{Resultados}

A partir del muestreo preliminar en los cultivos de café se lograron capturar ejemplares que fueron identificados taxonómicamente según las características morfológicas reportadas en adultos de $H$. hampei (Fig. 2), lo que permitió confirmar la presencia de broca en la zona de estudio y descartar la presencia de la falsa broca (Vega et al. 2015a; Alba-Alejandre et al. 2019). Las espinas anteriores en las tibias de las patas y antenas fueron importantes para identificar la especie, así como, la presencia de asperidades en el pronoto y estrías elitrales, esto permitió determinar que los cultivos de café en Coatepec, Veracruz si se encontraron infestados por el insecto.

En este muestreo preliminar se encontró que en los meses de septiembre y diciembre la población de insectos oscila en los 50 insectos, mientras que en el periodo del 1 al 15 de octubre se lograron capturar más de 150 insectos, siendo la mayor cantidad de adultos capturados, observando que se triplica su población lo que implica que hay un momento de invasión muy agresiva para las plantas de café (Fig. 3).

En los meses de septiembre, octubre y noviembre se pueden encontrar conteos cercanos a los 100 individuos lo que sugiere que el problema de la broca tiene relevancia en la región y no debe pasar desapercibida por los cafeticultores, por lo que debe considerarse como práctica común el colocar trampas para conocer la fluctuación de la población a través del tiempo en la zona. Se capturaron principalmente hembras adultas del barrenador de la baya del café, debido a que los machos no vuelan (Damon 2000; Dufour y Frérot 2008; Vega et al. 2015a). Después del muestreo preliminar se procedió a realizar el conteo de insectos utilizando las mezclas de los atrayentes propuestos en este trabajo, en el análisis de varianza se encontró que, si hay diferencia significativa entre el atrayente, el sitio y la fecha de muestreo $(\mathrm{p}<0,01)$. Mientras que en la interacción doble (Atrayente: Sitio), no hubo diferencia significativa $(\mathrm{p}=0,4947)$, lo que permite concluir que no influye el sitio de muestreo cuando se aplica el atrayente (Tabla 1). 


\section{2}

a)
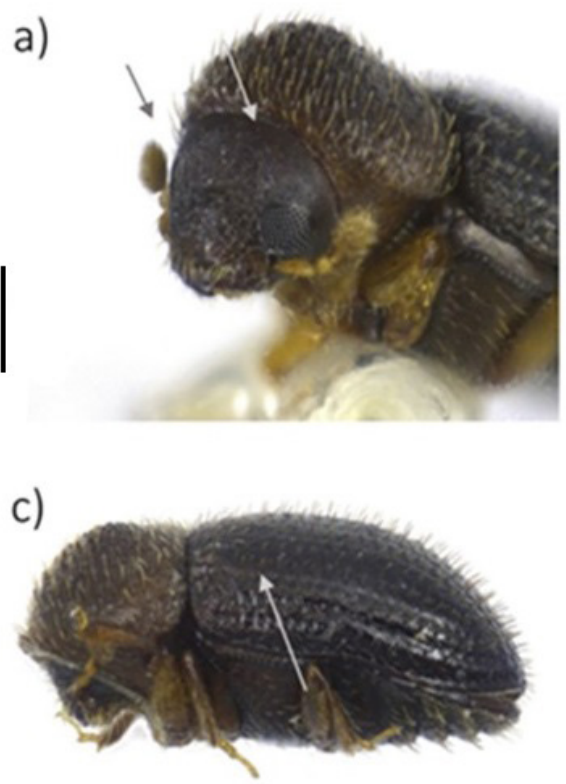

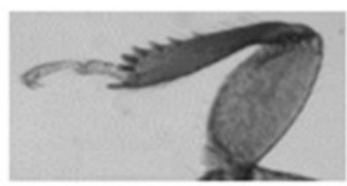

b)

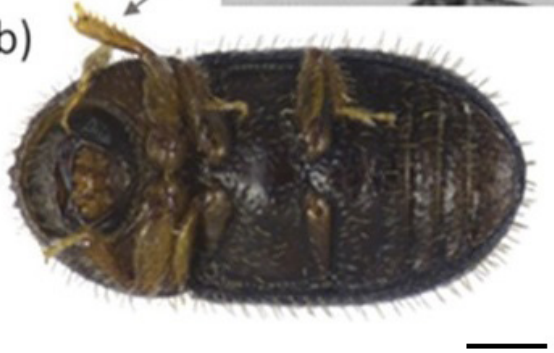

d)

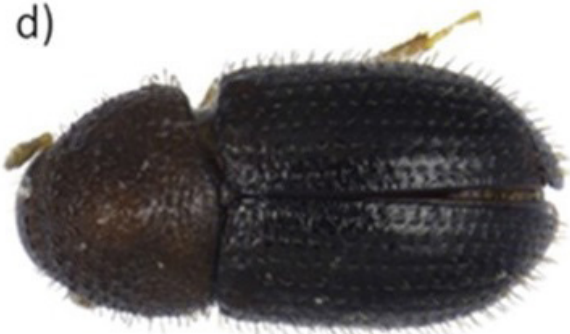

Figura 2. Características para la identificación de H. hampei: a) Cabeza con antenas y ojos. b) Adulto con asperidades en el pronoto y espinas en las tibias anteriores. c) Adulto con estrías parte lateral. d) Adulto con las estrías eritrales. Escala: $200 \mu \mathrm{m}$. / Characteristics for the identification of H. hampei: a) Head with antennae and eyes. b) Adult with asperities in the pronotum and spines in the anterior tibia. c) Adult with lateral part striae. d) Adult with erytral strials. Scale: $200 \mu \mathrm{m}$.

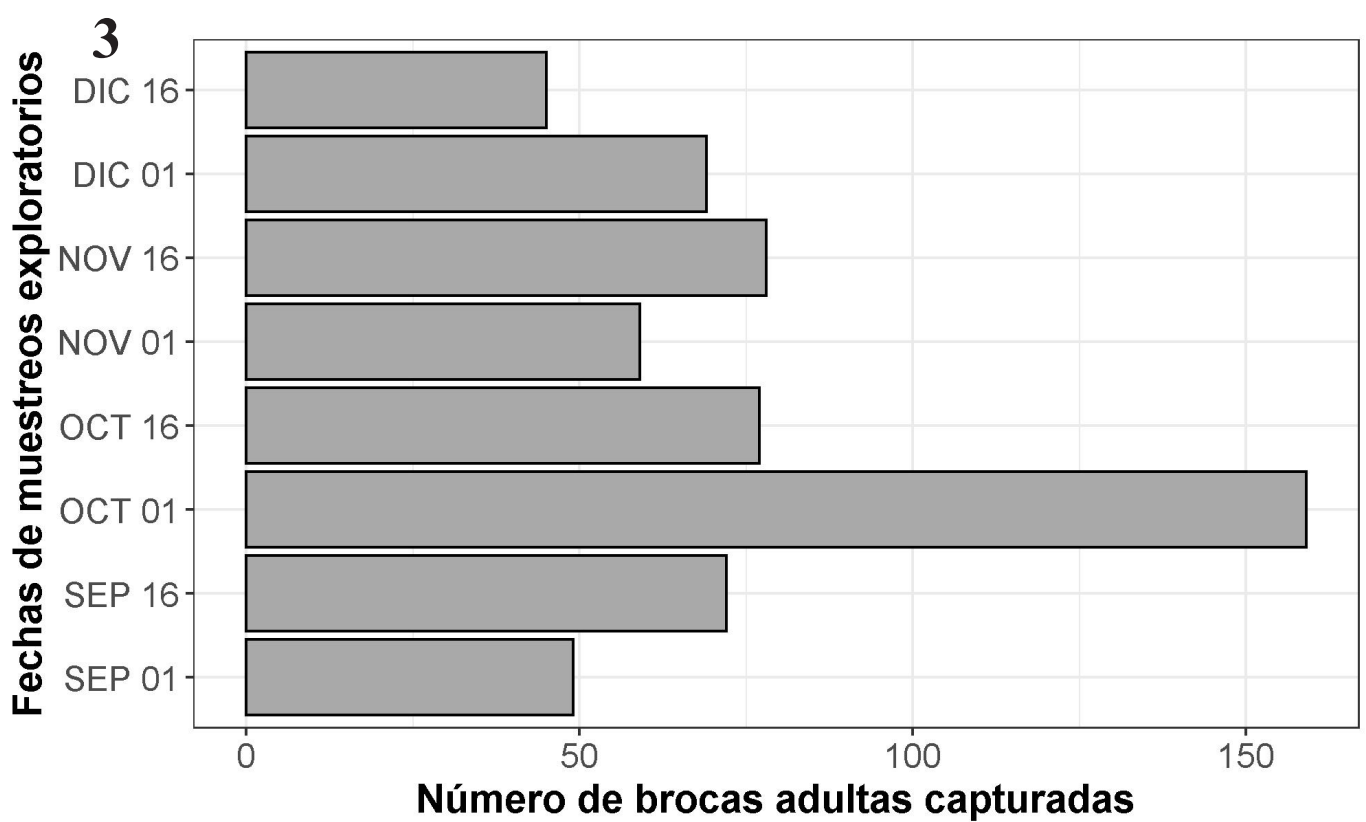

Figura 3. Número de insectos capturados en el muestreo preliminar. / Number of insects captured in the preliminary sampling. 
Tabla 1. Análisis de varianza para atrayentes, sitio de muestreo y fecha de captura de adultos de la broca del café. / Analysis of variance for attractants, sampling site and date of capture of adult coffee berry borer.

\begin{tabular}{lccccc}
\hline & GL & SC & CM & FC & $\operatorname{Pr}(>\mathrm{F})$ \\
\hline Atrayente & 5 & 259,877 & 51,975 & 48,650 & $0,0001^{* * *}$ \\
Sitio & 5 & 22,008 & 4,402 & 4,120 & $0,001^{* *}$ \\
Fecha & 20 & 158,023 & 7,901 & 7,396 & $0,0001^{* * *}$ \\
Atrayente: Sitio & 25 & 26,297 & 1,052 & 0,985 & 0,4947 \\
Residual & 94 & 100,425 & 1,060 & & \\
\hline
\end{tabular}

GL: Grados de libertad, SC: Suma de cuadrados, CM: Cuadrados medios, Fc: Estadística de Fisher, Pr: Nivel de significancia de la prueba.

De acuerdo al análisis de comparación de medias con la prueba de Tukey (Tabla 2), se observó que las mezclas de atrayentes que presentaron mayor número de capturas fueron las mezclas de etanol: vinagre de manzana (en promedio 136 insectos) y etanol: vinagre blanco (en promedio 98 insectos), ambos tratamientos presentan diferencias significativa entre sí pero podrían considerarse para la captura de H. hampei, ya que es importante el número de insectos que se logran capturar y además de que son más amigables con el ambiente y no tienen efectos tóxicos con la población como sucede con los insecticidas. Por otra parte, las mezclas de etanol con extractos etanólicos de las plantas estudiadas, no tuvieron diferencias entre ellas, podrían ser empleadas para la captura de la broca, aunque el efecto sería aproximadamente el 50\% menor a lo que se obtendría con las mezclas con los vinagres. En cuanto al tratamiento metanol- etanol 3:1 recomendado por el SENASICA y comúnmente usado para la captura, no tuvo un efecto significativo comparado con los tratamientos utilizados en esta investigación.

Tabla 2. Comparación de los diferentes atrayentes para la captura de H. hampei. / Comparison of the different attractants for the capture of H. hampei.

\begin{tabular}{lccc}
\hline \multicolumn{1}{c}{ Tratamiento } & Media & Error estándar de la media & Grupo \\
\hline Etanol: vinagre de manzana & 136,36 & 3,148966 & $\mathrm{a}$ \\
Etanol: vinagre blanco & 98,24 & 2,453173 & $\mathrm{~b}$ \\
Etanol: extracto etanólico de albahaca & 44,48 & 1,223456 & $\mathrm{C}$ \\
Etanol: extracto etanólico de ruda & 56,68 & 1,230757 & $\mathrm{C}$ \\
Etanol: extracto etanólico de romero & 42,92 & 1,214683 & $\mathrm{C}$ \\
Metanol: Etanol (testigo) & 8,24 & 0,158021 & $\mathrm{~d}$ \\
\hline
\end{tabular}

\section{Discusión}

Las trampas con atrayentes se han utilizado con éxito para el muestreo de insectos plaga porque ahorran tiempo, son fáciles de usar, permiten la captura y el confinamiento de los insectos (Fernandes et al. 2015). Cabe hacer mención que los diferentes atrayentes utilizados en este estudio no han sido reportados en la literatura, aunque se han hecho ya algunos estudios con mezclas de alcoholes como el etanol y metanol en diferentes proporciones e incluso con aguardiente de agave cocuy, aguardiente de caña de azúcar, y vinagre de pulpa de café (Fernández y Cordero 2005). 
Por otra parte, la selección de las trampas se basó en su facilidad para prepararse y utilizarse en las plantas de café lo que se reflejó en la eficacia de la captura, otros autores han utilizado el trampeo con diversos atrayentes con la misma finalidad (Damon 2000; Bacca et al. 2008; Fernández y Cordero 2005). Las trampas elaboradas con botellas de PET (botellas de tereftalato de polietileno) han sido utilizadas para colocar los atrayentes con resultados satisfactorios (Mathieu et al. 1999; Dufour y Frérot 2008; Fernandes et al. 2015), ya que las hembras responden muy bien a estímulos olfativos (Mathieu et al. 2001).

Los resultados obtenidos por los anteriores autores coinciden con los obtenidos en este trabajo, donde se utilizó el mismo material, pero con diferentes mezclas que son amigables con el ambiente y menos tóxicas para el ser humano. De manera tradicional, se ha utilizado la mezcla sinérgica de metanol-etanol, como el más eficiente para estudiar la broca del café en México (Gutiérrez-Martínez y Ondarza 1996), El Salvador (Dufour y Frérot 2008), Brasil (Silva et al. 2006; Uemura-Lima et al. 2010; Pereira et al. 2012) y Hawái (Messing 2012).

El etanol es un solvente volátil emitido por los granos de café, no es producido por la planta, pero ha funcionado eficientemente. El metanol es tóxico para el humano y puede dañar por ingestión, inhalación o absorción cutánea con variadas consecuencias, lo que representa un peligro para los productores que lo utilizan en campo (Clary 2013), aunado a lo anterior Messing (2012), publicó que uno de los principales problemas que enfrenta el agricultor es el uso de metanol, ya que no está ampliamente disponible el solvente en las regiones rurales y además la cantidad de metanol que se requiere es en pequeñas cantidades puesto que la mayoría de las fincas cafetaleras no son tan extensas y además tienen una producción que depende de las condiciones climatológicas.

En la búsqueda de nuevas sustancias para la captura de la broca del café, se han realizado también otros trabajos empleando diversos semioquímicos, con la finalidad de evaluar la eficacia de otros atrayentes, además de que se ha reportado el uso de extractos de granos de café maduros y cafeína disuelta en etanol (Gutiérrez-Martínez y Ondarza 1996); metanol-etanol con aceite de café (Silva et al. 2006); cafeína, café en polvo verde, filtrado de café recién molido y extracto de broca del café (Dufour y Frérot 2008) e incluso se han realizado estudios de compuestos volátiles que son liberados por las bayas de café como metilciclohexano, nonano, etilbenceno, (R)-limoneno, (S)-limoneno, 1-octen-3-ol y 3-etil-4-metilpentanol para conocer si hay respuestas electrofisiológicas que afecten a las antenas de H. hampei (Mendesil et al. 2009; Cruz y Malo 2013).

Los estudios sobre las especies Coffea arabica L. y Coffea canephora, Pierre ex A. Froehner, revelaron la preferencia de $H$. hampei por varios compuestos odorantes (Guerreiro y Mazzafera 2003). Por lo tanto, probar la atracción de H. hampei con diferentes compuestos de plantas como los evaluados en esta investigación, tiene un gran potencial para ser una alternativa en el manejo integrado de plagas, ya que las trampas permiten hacer la colecta, y se pueden establecer con precisión los datos sobre la densidad de la población de plagas importantes, y así tener una mejor toma de decisiones que sean más sostenibles y exitosas (Fernandes et al. 2015).

El conocimiento de la dispersión de insectos es importante para determinar la ubicación de trampas con atrayentes en programa de seguimiento y captura masiva de broca, de manera que sea más eficiente su control (Aristizábal et al. 2015). En este estudio, se vio por primera vez la factibilidad de usar trampas con mezclas que son económicamente viables para los productores y que al utilizarlos con trampas artesanales pueden resultar en una estrategia innovadora, segura y sostenible para la captura de la broca del café.

\section{Conclusión}

El uso de trampas artesanales con etanol y vinagre para la captura de adultos de broca se presenta como una alternativa de bajo costo, que es factible para ser utilizada 
exitosamente por los productores en programas de manejo integrado de este insecto plaga en el cultivo de café. En este estudio se encontró que los mejores tratamientos que sirvieron como atrayentes en trampas artesanales para la captura de H. hampei fueron; etanol con vinagre de manzana y etanol con vinagre blanco a una proporción 1:1, esto contribuirá al desarrollo de nuevas mezclas volátiles que pueden proveer una estrategia sustentable para la captura de la broca del café.

\section{Agradecimientos}

A los cafeticultores de la comunidad Bella Esperanza, del Municipio de Coatepec, Veracruz, México.

\section{Literatura Citada}

Alba-Alejandre, I., Alba-Tercedor, J. y Vega, F.E. (2018) Observing the devastating coffee berry borer (Hypothenemus hampei) inside the coffee berry using micro-computed tomography. Scientific Reports, 8: 1-9.

Alba-Alejandre, I., Alba-Tercedor, J. y Vega, F.E. (2019) Anatomical study of the coffee berry borer (Hypothenemus hampei) using micro-computed tomography. Scientific Reports, 9(1): 1-16.

Aristizábal, L.F., Jiménez, M., Bustillo, A.E., Trujillo, H.I. y Arthurs, S.P. (2015) Monitoring coffee berry borer, Hypothenemus hampei (Coleoptera: Curculionidae), populations with alcohol-baited funnel traps in coffee farms in Colombia. Florida Entomologist, 98(1): 381-383.

Aristizábal, L.F., Bustillo, A.E. y Arthurs, S.P. (2016) Integrated pest management of coffee berry borer: strategies from Latin America that could be useful for coffee farmers in Hawaii. Insects, 7(1): 6.

Bacca, T., Lima, E.R., Picanço, M.C., Guedes, R.N.C. y Viana, J.H.M. (2008) Sampling plan for the coffee leaf miner Leucoptera coffeella with sex pheromone traps. Journal of Applied Entomology, 132: 430-438.

Bruce, T.J.A., Wadhams, L.J. y Woodcock, C.M. (2005) Insect host location: a volatile situation. Trends in Plant Science, 10(6): 269-274.

Clary, J.J. (2013) Human toxicity. Pp: 47-72. In: Clary, J.J. (Ed). The toxicology of methanol. Ed. John Wiley and Sons. New Jersey.

Cruz, E.N y Malo, EA. (2013) Chemical analysis of coffee berry volatiles that elicit an antennal response from the coffee berry borer Hypothenemus hampei. Journal of the Mexican Chemical Society, 57(4): 321-327.

Damon, A. (2000) A review of the biology and control of the coffee berry borer, Hypothenemus hampei (Coleoptera: Scolytidae) (Coleoptera: Scolytidae). Bulletin of Entomological Research, 90(6): 453-465.

Dufour, B.P. y Frérot, B. (2008) Optimization of coffee berry borer, Hypothenemus hampei Ferrari (Col., Scolytidae), mass trapping with an attractant mixture. Journal of Applied Entomology, 132(7): 591-600.

Fernandes, F.L., Picanço, M.C., Fernandes, M.E.S., Dângelo, R.A.C., Souza, F.F. y Guedes, R.N.C. (2015) A new and highly effective sampling plan using attractant-baited traps for the coffee berry borer (Hypothenemus hampei). Journal of Pest Science, 88(2): 289-299.

Fernández, S. y Cordero, J. (2005) Evaluación de atrayentes alcohólicos en trampas artesanales para el monitoreo y control de la broca del café, Hypothenemus hampei (Ferrari). Bioagro, 17(3): 143-148.

Gómez, J., Chávez, B.Y., Castillo, A., Valle, F.J. y Vega, F.E. (2015) The coffee berry borer (Coleoptera: Curculionidae): How many instars are there? Annals of the Entomological Society of America, 108(3): 311-315. 
Green, P.W., Davis, A.P., Cossé, A.A. y Vega, F.E. (2015) Can coffee chemical compounds and insecticidal plants be harnessed for control of major coffee pests? Journal of Agricultural Food Chemistry, 63(43): 9427-9434.

Guerreiro, O. y Mazzafera, P. (2003) Caffeine and resistance of coffee to the berry borer Hypothenemus hampei (Coleoptera:Scolytidae). Journal of Agriculture and Food Chemistry, 51(24): 6987-6991.

Gutiérrez-Martínez, A. y Ondarza, R.N. (1996) Kairomone effect of extracts from Coffea canephora over Hypothenemus hampei (Coleoptera: Scolytidae). Environmental Entomology, 25(1): 96-100.

Infante, F. (2018) Pest management strategies against the coffee berry borer (Coleoptera: Curculionidae: Scolytinae). Journal of Agricultural and Food Chemistry, 66(21): 5275-5280.

Mathieu, F., Brun, L.O., Frerot, B., Suckling, D.M. y Frampton, C. (1999) Progression in field infestation is linked with trapping of coffee berry borer, Hypothenemus hampei (Coleoptera: Scotytidae). Journal of Applied Entomology, 123: 535-540.

Mathieu, F., Gaudichon, V., Brun, L.O. y Frérot, B. (2001) Effect of physiological status on olfactory and visual responses of female Hypothenemus hampei during host plant colonization. Physiological Entomology, 26: 189-193.

Mendesil, E., Bruce, T.J., Woodcock, C.M., Caulfield, J.C., Seyoum, E. y Pickett, J.A. (2009) Semiochemicals used in host location by the coffee berry borer, Hypothenemus hampei. Journal of Chemistry and Ecology, 35(8): 944-950.

Messing, R.H. (2012) The coffee berry borer (Hypothenemus hampei) invades Hawaii: Preliminary investigations on trap response and alternate hosts. Insects, 3(3): 640-652.

Pereira, A.E., Vilela, E.F., Tinoco, R.S., De Lima, J.O.G., Fantine, A.K., Morais, E.G. y França, C.F. (2012) Correlation between numbers captured and infestation levels of the coffee berry-borer, Hypothenemus hampei: A preliminary basis for an action threshold using baited traps. International Journal of Pest Management, 58: 183-190.

Prates, H.S. (1969) Observaçoes preliminares de atraçao da broca do café Hypothenemus hampei (Ferrari, 1867), a extratos de frutos do cafeeiro (cereja e verde). O Solo, 61: 13-14.

Rodríguez, D., Cure, J.R., Gutierrez, A.P. y Cotes, J.M. (2017) A coffee agroecosystem model: III. Parasitoids of the coffee berry borer (Hypothenemus hampei). Ecological Modelling, 363: 96-110.

SENASICA (2008) Apéndice de manejo integrado de la broca del café en México (Manual Técnico-Operativo de la campaña contra la broca del café). Dirección de Protección Fitosanitaria. México. Pp. 66. http://publico.senasica.gob.mx/includes/asp/ download.asp? IdDocumento $=3888 \&$ IdUrl $=43058 \&$ objeto $=\mathrm{P} \%$ E1gina $\&$ IdObjetoBase $=4$ $647 \&$ down $=$ true

Silva, F.C.D., Ventura, M.U. y Morales, L. (2006) Capture of Hypothenemus hampei Ferrari (Coleoptera: Scolytidae) in response to trap characteristics. Scientia Agricola, 63(6): 567571.

Uemura-Lima, D.H., Ventura, M.U., Mikami, A.Y., Da Silva, F.C. y Morales, L. (2010) Responses of coffee berry borer, Hypothenemus hampei (Ferrari) (Coleoptera: Scolytidae) to vertical distribution of methanol: ethanol traps. Neotropical Entomology, 39(6): 930-933.

Vega, F.E., Infante, F. y Johnson, A.J. (2015a) The genus Hypothenemus, with emphasis on H. hampei, the coffee berry borer. Pp. 427-494. In: Vega, F. and Hofstetter, R. (Eds). Bark beetles: Biology and ecology of native and invasive species. 1st edition. Elsevier Academic Press. London.

Vega, F.E., Bauchan, G., Infante, F., Valdéz-Carrasco, J.M. y Beaver, R. (2015) Visualizing the mesothoracic spiracles in a bark beetle: Hypothenemus hampei (Coleoptera: Curculionidae). Annals of the Entomological Society of America, 108(3): 446-448. 